\title{
Global Bifurcation for the Whitham Equation
}

\author{
M. Ehrnström ${ }^{1}$, H. Kalisch ${ }^{2}$. \\ ${ }^{1}$ Department of Mathematical Sciences, Norwegian University of Science and Technology \\ 7491 Trondheim, Norway \\ ${ }^{2}$ Department of Mathematics, University of Bergen \\ Postbox 7800, 5020 Bergen, Norway
}

\begin{abstract}
We prove the existence of a global bifurcation branch of $2 \pi$-periodic, smooth, traveling-wave solutions of the Whitham equation. It is shown that any subset of solutions in the global branch contains a sequence which converges uniformly to some solution of Hölder class $C^{\alpha}, \alpha<\frac{1}{2}$. Bifurcation formulas are given, as well as some properties along the global bifurcation branch. In addition, a spectral scheme for computing approximations to those waves is put forward, and several numerical results along the global bifurcation branch are presented, including the presence of a turning point and a 'highest', cusped wave. Both analytic and numerical results are compared to traveling-wave solutions of the KdV equation.
\end{abstract}

Keywords and phrases: Whitham equation, global bifurcation, traveling waves, spectral projection, cosine transform

Mathematics Subject Classification: 35Q53, 35C07, 45K05, 65M70, 76B15

\section{Introduction}

The Whitham equation,

$$
\eta_{t}+\frac{3}{2} \frac{c_{0}}{h_{0}} \eta \eta_{x}+K_{h_{0}} * \eta_{x}=0,
$$

combines a generic nonlinear quadratic term with the exact linear dispersion relation for surface water waves on finite depth. Here, the kernel $K_{h_{0}}:=\mathcal{F}^{-1}\left(c_{h_{0}}\right)$ is the inverse Fourier transform of the phase speed

$$
c_{h_{0}}(\xi):=\sqrt{\frac{g \tanh \left(\xi h_{0}\right)}{\xi}}
$$

for the linearized water-wave problem; the constants $g, h_{0}$ and $c_{0}:=\sqrt{g h_{0}}$ denote, respectively, the gravitational constant of acceleration, the undisturbed water depth, and the limiting long-wave speed. The function $\eta(t, x)$ describes the deflection of the fluid surface from the rest position at a point $x$ at time $t$ [19]. The Whitham equation was introduced by Whitham (see [18]) as an alternative to the Korteweg-de Vries (KdV) equation, which describes the evolution of predominantly uni-directional, small-amplitude surface waves in shallow water $[2,8,16]$. The $\mathrm{KdV}$ equation appears from (1.1) if one replaces $c_{h_{0}}(\xi)$ with its second-order approximation at $\xi=0$,

$$
c_{0}-\frac{1}{6} c_{0} h_{0}^{2} \xi^{2},
$$

\footnotetext{
${ }^{*}$ Corresponding author. E-mail: henrik.kalisch@math.uib.no
} 
and this explains the main motivation behind the Whitham equation. In particular, since $\xi=\frac{2 \pi}{\lambda}$ may be interpreted as a wave number, $\lambda$ being a typical wavelength, one sees from (1.3) that the KdV equation is a poor approximation to (1.2) for large wave numbers.

The Whitham equation (1.1) with the kernel (1.2) has some very interesting mathematical features. First of all, it is generically nonlocal, making pointwise estimates difficult. Moreover, $c_{h_{0}}(\xi)$ has slow decay, and the kernel $K_{h_{0}}$ is singular (it blows up at $x=0$ ). This makes the Whitham equation in some important respects different from many other equations of the form (1.1). One example is the possibility of singularities in solutions: the Whitham equation features (phenomenological) wave-breaking [15] i.e. bounded solutions with unbounded derivatives - and is conjectured to admit solutions with cusps [18].

In this paper we consider steady solutions of the Whitham equation, i.e., traveling-wave solutions characterized by a constant speed and shape. The existence of smooth, small-amplitude, periodic travelingwave solutions was established in [10], and their properties numerically investigated. The numerical calculations pursued in that investigation indicate a global branch of solutions approaching a 'highest', cusped, wave - as well as a sequence of periodic waves converging to a solitary wave. An analytic proof of the latter fact is given in [9], for small waves, using a variational approach developed mainly in $[5,12]$. The aim of this note is: (i) to place the Whitham equation in a general functional-analytic framework, in which the bifurcation theory for that and many related equations is quite natural; (ii) to extend the main local bifurcation branch found in [10] to a global one; and (iii) to provide a spectral scheme for efficient calculation of Whitham waves. In addition, we prove some convergence results along the global branch of solutions, and several numerical calculations are given, complementing the more abstract exact theory. In particular, numerical evidence is given for a turning point along the global bifurcation branch, as well as additional calculations supporting the existence of a cusped wave at the end of the bifurcation branch.

Judging from the forms of the $\mathrm{KdV}$ and Whitham equations, one would expect several similarities for small waves of small wavelength (one such convergence result is given in [9]). To illustrate this, we perform a comparative local bifurcation analysis for the two equations, analytically and numerically. Although the solutions arise in the same manner from a curve of vanishing solutions, they develop differently along the corresponding bifurcation branches. We show that the Whitham solutions are all smooth and subcritical (their normalized wave speed is uniformly bounded away from unity), and that they converge uniformly to a wave of $C^{\alpha}$-regularity, $\alpha<\frac{1}{2}$. The intricate form of the Whitham kernel has yet hindered us from proving that the limiting wave is cusped and non-trivial. To see the difficulty, note that the Whitham kernel $K_{h_{0}}$ is not easily seen to be positive (cf., e.g., [11]).

This is the disposition of the paper: in Section 2 we normalize the equation for traveling wavesolutions and briefly discuss Fourier multipliers on Hölder spaces. Section 3 contains a functional-analytic formulation of the problem, and local bifurcation theory for the Whitham and the KdV equations. Section 4 is the main analytic section, in which we state and prove the global results. The sections 5 and 6 are devoted to the numerical analysis. In Section 5 we describe a spectral scheme for the Whitham equation, and use it to calculate wave profiles. Finally, Section 6 takes a look at the shape of the bifurcation branches, and provides a numerical comparison of the KdV and Whitham waves.

\section{Some preliminaries}

Throughout the paper, the operator $\mathcal{F}$ will denote the extension to the space of tempered distributions $\mathcal{S}^{\prime}(\mathbb{R})$ of the Fourier transform

$$
\mathcal{F}(f)(\xi):=\int \hat{f}(x) \exp (-i x \xi) d x
$$

on the Schwartz space $\mathcal{S}(\mathbb{R})$, with inverse $\mathcal{F}^{-1}(f)(x):=\frac{1}{2 \pi} \int \hat{f}(\xi) \exp (i x \xi) d \xi$. 


\subsection{Traveling waves.}

By considering steady solutions with wave speed $c>0$,

$$
\varphi(x-c t):=\eta(t, x),
$$

the left-hand side of (1.1) may be integrated to $-c \varphi+\frac{3 c_{0}}{4 h_{0}} \varphi^{2}+K_{h_{0}} * \varphi$. In the following, we keep with the convention in denoting the steady variable $x-c t$ again by $x$. The scalings $\frac{3}{4 h_{0}} \varphi \mapsto \varphi$ and $x \mapsto h_{0} x$ then yield the normalized equation

$$
-\mu \varphi+\varphi^{2}+K * \varphi=0
$$

where $\mu:=c / c_{0}$ is the non-dimensional wave speed, and we have set the constant of integration equal to zero (see Remark 2.1 below for a discussion of this). The symbol of the convolution operator in (2.1) is given by

$$
\mathcal{F}(K)(\xi):=\left(\frac{\tanh (\xi)}{\xi}\right)^{1 / 2}
$$

for the Whitham equation. The corresponding Fourier multiplier for the KdV equation has the form $1-\frac{1}{6} \xi^{2}$, and the equation reads

$$
(1-\mu) \varphi+\varphi^{2}+\frac{1}{6} \varphi^{\prime \prime}=0
$$

Any solution of (2.1) with the kernel given by (2.2) will be called a Whitham solution, and any solution of (2.3) will be called a $K d V$ solution. We shall be dealing only with $2 \pi$-periodic solutions.

Remark 2.1. The normalization $B=0$ in

$$
-\mu \varphi+\varphi^{2}+K * \varphi=B
$$

cf. (2.1), is a choice of convenience: both the Whitham and KdV equations are invariant under the Galilean transformation

$$
\varphi \mapsto \varphi+\gamma, \quad \mu \mapsto \mu+2 \gamma, \quad B \mapsto B+\gamma(1-\mu-\gamma),
$$

for any $\gamma \in \mathbb{R}$. As an alternative to letting $B=0$ one may therefore consider waves of vanishing mean amplitude.

\subsection{Fourier multipliers on Hölder spaces}

The analysis given in this paper relies on certain properties of Fourier multiplier operators given by classical symbols, and we briefly recall some of those. A smooth, real-valued function $a$ on $\mathbb{R}$ is said to be in the symbol class $S^{m}$ if for some constant $c>0$ and any non-negative integer $k$, the estimate

$$
\left|\partial_{\xi}^{k} a(\xi)\right| \leq c(1+|\xi|)^{m-k}
$$

holds. Analyzing the action of such operators on the Sobolev spaces $H^{s}$ is straightforward owing to Parseval's formula. However, the analysis is somewhat more subtle if classes of continuously differentiable functions or other Banach spaces are considered. Although there exist natural scales of function spaces for this class of operators (cf. [17]) we have chosen to work with the classical spaces $C_{\text {even }}^{s}(\mathbb{S}), s>0$, consisting of $2 \pi$-periodic even functions that are $\lfloor s\rfloor$ times continuously differentiable with the $\lfloor s\rfloor$ th derivative bounded and continuous, and Hölder continuous with Hölder exponent $s-\lfloor s\rfloor$ for $s \notin \mathbb{Z}$. We then have the following lemma.

Lemma 2.2 ([1]). Fix $m \in \mathbb{R}, a \in S^{m}$, and let $s \in \mathbb{R}_{+} \backslash \mathbb{Z}$ be a non-integral non-negative real number. Then

$$
a(\mathrm{D}) \in \mathcal{L}\left(C(\mathbb{S})^{s+m}, C(\mathbb{S})^{s}\right)
$$

is a bounded linear operator of order $-m$. 
Remark 2.3. If one extends instead the family of Hölder spaces $C^{s}$, for $s \notin \mathbb{Z}$, with the so-called Zygmund spaces for integral values of $s$, one attains a scale of spaces for which Lemma 2.2 is valid also for integral values of $s$ (for more information on the relations between these different function spaces, see [17]). The introduction of Zygmund spaces is, however, not necessary to obtain the results in this paper.

\section{Comparative local bifurcation theory}

From now on, let $\frac{1}{2}<\alpha<1$. We consider $C_{\text {even }}^{\alpha}(\mathbb{S})$, the space of even and $\alpha$-Hölder continuous real-valued functions on the unit circle $\mathbb{S}$. The Whitham equation contains a generic nonlocal smoothing operator in the form of the Fourier multiplier $(\tanh (\xi) / \xi)^{1 / 2}$, whereas the corresponding $\mathrm{KdV}$ multiplier $1-\frac{1}{6} \xi^{2}$ is a second order differential operator. This difference may be easily overcome: rewriting the KdV equation in non-local, smoothing, form enables a similar treatment of the two equations. To illustrate how the analysis used for the Whitham equation can be applied to a larger class of equations, a comparative local bifurcation analysis is performed for the Whitham and the KdV equation (the KdV equation being a cardinal example of dispersive evolution equations and the Whitham equation's closest kin).

Theorem 3.1 (Functional-analytic formulation). Fix $\alpha>\frac{1}{2}$ and let $\mu>0$. The solutions in $C_{\text {even }}^{\alpha}(\mathbb{S})$ of the Whitham equation (2.1), and those of the KdV equation (2.3), coincide with the kernel of an analytic operator $\mathrm{F}: C_{\text {even }}^{\alpha}(\mathbb{S}) \times \mathbb{R}_{>0} \rightarrow C_{\text {even }}^{\alpha}(\mathbb{S})$ given by

$$
\mathrm{F}(\varphi, \mu):=\mu \varphi-\mathrm{L}(\mu) \varphi+\mathrm{N}(\varphi, \mu),
$$

where $\mathrm{L}(\mu)$ is bounded, linear and compact, and

$$
\mathrm{N}_{W}(\varphi, \mu)=-\varphi^{2}, \quad \mathrm{~N}_{K d V}(\varphi, \mu)=-\mathrm{L}(\mu) \varphi^{2},
$$

fulfills $\mathrm{D}_{\varphi} \mathrm{N}[0, \mu]=0$. Thus $\mathrm{D}_{\varphi} \mathrm{F}[0, \mu]$ is Fredholm of index 0 . In the case of the Whitham equation the operators $\mathrm{L}$ and $\mathrm{N}$ are independent of $\mu$.

Proof. We put the KdV equation in the form

$$
\mu \varphi=\left(1-\frac{1}{6 \mu} \partial_{x}^{2}\right)^{-1}\left(\varphi+\varphi^{2}\right)
$$

where $\left(1-\tau^{2} \partial_{x}^{2}\right)^{-1} f:=\frac{1}{2 \tau} \exp \left(-\frac{|\cdot|}{\tau}\right) * f$ is the inverse of $1-\tau^{2} \partial_{x}^{2}$ on $\mathcal{S}^{\prime}(\mathbb{R})$, the Schwartz space of tempered distributions on $\mathbb{R}$. Define

$$
\mathrm{L}_{\mathrm{W}}:=K * \quad \text { and } \quad \mathrm{L}_{\mathrm{KdV}}:=\left(1-\frac{1}{6 \mu} \partial_{x}^{2}\right)^{-1} .
$$

For functions $f \in C_{\text {even }}^{\alpha}(\mathbb{S})$ with $\alpha>1 / 2$ one has

$$
f(x) \equiv \sum_{k \geq 0} a_{k} \cos (k x) \quad \text { and } \quad \sum_{k \geq 0}\left|a_{k}\right|<\infty,
$$

meaning that $C_{\text {even }}^{\alpha}(\mathbb{S})$ is a subalgebra of the Wiener algebra of $2 \pi$-periodic functions with absolutely converging Fourier series [13]. For such functions rudimentary calculations reduce (3.1) to the intuitive formulas

$$
\begin{aligned}
\mathrm{L}_{\mathrm{W}} f(x) & :=\sum_{k \geq 0} a_{k}\left(\frac{\tanh (k)}{k}\right)^{1 / 2} \cos (k x), \\
\mathrm{L}_{\mathrm{KdV}}(\mu) f(x) & :=\sum_{k \geq 0} \frac{a_{k}}{1+\frac{1}{6 \mu} k^{2}} \cos (k x),
\end{aligned}
$$


where for the Whitham equation one must use the fact that $K \in L^{1}(\mathbb{R})$ (for details on $K$ and its symbol, see [10]). The Fourier multiplier symbols in (3.2) and (3.3) belong to the symbol classes $S^{-1 / 2}(\mathbb{R})$ and $S^{-2}(\mathbb{R})$, and $(3.2)$ and $(3.3)$ are therefore bounded linear operators $C_{\text {even }}^{\alpha}(\mathbb{S}) \rightarrow C_{\text {even }}^{\alpha+s}(\mathbb{S})$ for $s=1 / 2$ and $s=2$, respectively, see Lemma 2.2. Each of them is also invertible with bounded linear inverse $\mathrm{L}^{-1}: C_{\text {even }}^{\alpha+s}(\mathbb{S}) \rightarrow C_{\text {even }}^{\alpha}(\mathbb{S})$ for the same values of $s$. Due to the compactness of the embedding $C_{\text {even }}^{\beta}(\mathbb{S}) \hookrightarrow$ $C_{\text {even }}^{\alpha}(\mathbb{S}), \beta>\alpha$, both operators are compact on $C_{\text {even }}^{\alpha}(\mathbb{S})$. We may thus define mappings $\mathrm{F}_{\mathrm{w}}$ and $\mathrm{F}_{\mathrm{KdV}}: C_{\text {even }}^{\alpha}(\mathbb{S}) \times \mathbb{R}_{>0} \rightarrow C_{\text {even }}^{\alpha}(\mathbb{S})$ by

$$
\begin{aligned}
\mathrm{F}_{\mathrm{W}}(\varphi, \mu) & :=\mu \varphi-\mathrm{L}_{\mathrm{W}} \varphi-\varphi^{2}, \\
\mathrm{~F}_{\mathrm{KdV}}(\varphi, \mu) & :=\mu \varphi-\mathrm{L}_{\mathrm{KdV}}(\mu) \varphi-\mathrm{L}_{\mathrm{KdV}}(\mu) \varphi^{2} .
\end{aligned}
$$

In both cases $F$ is analytic in both its arguments $\left(\mathrm{L}_{\mathrm{KdV}}(\mu)\right.$ is the inverse of $1-\frac{1}{6 \mu} \partial_{x}^{2}$, which is analytic in $\mu$ ). We also have $F(0, \mu)=0$, and the linearization $\mathrm{D}_{\varphi} \mathrm{F}[0, \mu]=\mu \mathrm{id}-\mathrm{L}$ is Fredholm of index 0 (it is a continuous perturbation of the identity by a compact operator).

\subsection{Local bifurcation}

Corollary 3.2 (Local bifurcation, Whitham).

(i) Subcritical bifurcation. For each integer $k \geq 1$, there exist $\mu_{k}:=(\tanh (k) / k)^{1 / 2}$ and a local, analytic curve

$$
\varepsilon \mapsto(\varphi(\varepsilon), \mu(\varepsilon)) \in C_{\text {even }}^{\alpha}(\mathbb{S}) \times(0,1)
$$

of nontrivial $2 \pi / k$-periodic Whitham solutions with $\mathrm{D}_{\varepsilon} \varphi(0)=\cos (k x)$ that bifurcates from the trivial solution curve $\mu \mapsto(0, \mu)$ at $(\varphi(0), \mu(0))=\left(0, \mu_{k}\right)$. In a neighborhood of the bifurcation point $\left(0, \mu_{k}\right)$ these are all nontrivial solutions of $\mathrm{F}_{\mathrm{W}}(\varphi, \mu)=0$ in $C_{\text {even }}^{\alpha}(\mathbb{S}) \times(0,1)$, and there are no other bifurcation points $\mu>0, \mu \neq 1$, for solutions in $C_{\text {even }}^{\alpha}(\mathbb{S})$.

(ii) Transcritical bifurcation. At $\mu=1$ the trivial solution curve $\mu \mapsto(0, \mu)$ intersects the curve $\mu \mapsto(\mu-1, \mu)$ of constant solutions $\varphi=\mu-1$; together these constitute all solutions in $C_{\text {even }}^{\alpha}(\mathbb{S})$ in a neighborhood of $(\varphi, \mu)=(0,1)$.

Proof. The fact that $\mathrm{D}_{\varphi} \mathrm{F}_{\mathrm{W}}[0, \mu]=\mu \mathrm{id}-\mathrm{L}_{\mathrm{W}}$ is Fredholm of index 0 and the formula (3.2) show that $\mu_{k}$ are all simple eigenvalues of $\mathrm{L}_{\mathrm{W}}$, and that no other eigenvalues $\mu>0$ exist. The assertion then follows from the analytic version of the Crandall-Rabinowitz theorem for bifurcation from a simple eigenvalue [6, Thm 8.4.1]. The fact that the solutions are $2 \pi / k$-periodic can be seen by restricting attention to the subspaces $\left\{\varphi \in C_{\text {even }}^{\alpha}(\mathbb{S}): \varphi\right.$ is $2 \pi / k$-periodic $\}$, and, corresponding to the case $k=0$, it is instantly verified that $\varphi=\mu-1$ is a solution. By uniqueness, this family must therefore constitute the local bifurcation curve at $\mu=1$. Since for all other $\mu>0$ the linearization $\mathrm{D}_{\varphi} \mathrm{F}_{\mathrm{W}}[0, \mu]$ is Fredholm of index zero with trivial kernel, it is a consequence of the implicit function theorem that the vanishing solution is, locally, the unique solution in $C_{\text {even }}^{\alpha}(\mathbb{S})$.

Remark 3.3. A more detailed proof of local bifurcation in a somewhat larger space, for $k=1$, but with arbitrary wavelength, was given in [10].

For the KdV equation the change of variables

$$
\varphi \mapsto k^{2} \varphi(k \cdot), \quad \mu \mapsto k^{2}(\mu-1)+1,
$$

means that the local bifurcation analysis can be reduced to the case $k=1$.

Corollary 3.4 (Local bifurcation, KdV). Let $\mu^{*}:=5 / 6$. A local, analytic curve $\varepsilon \mapsto(\varphi(\varepsilon), \mu(\varepsilon)) \in$ $C_{\text {even }}^{\alpha}(\mathbb{S}) \times \mathbb{R}_{>0}$ of nontrivial KdV solutions with $\mathrm{D}_{\varepsilon} \varphi(0)=\cos (x)$ bifurcates from the trivial solution curve at $\left(0, \mu^{*}\right)$. 
Proof. We have that

$$
\mathrm{D}_{\varphi} \mathrm{F}_{\mathrm{KdV}}[0, \mu] \varphi=\sum_{k \geq 0} a_{k}\left(\mu-\frac{6 \mu}{6 \mu+k^{2}}\right) \cos (k x) .
$$

Therefore, $\operatorname{ker}\left(\mathrm{D}_{\varphi} \mathrm{F}_{\mathrm{KdV}}\left[0, \mu^{*}\right]\right)=\operatorname{span}(\cos (x))$ and

$$
\mathrm{D}_{\varphi \mu}^{2} \mathrm{~F}_{\mathrm{KdV}}\left[0, \mu^{*}\right] \cos (x)=\frac{5}{6} \cos (x) \notin \operatorname{ran}\left(\mathrm{D}_{\varphi} \mathrm{F}_{\mathrm{KdV}}\left[0, \mu^{*}\right]\right) .
$$

As in the proof of Corollary 3.2, since $\mathrm{D}_{\varphi} \mathrm{F}_{\mathrm{KdV}}\left[0, \mu^{*}\right]$ is Fredholm of index 0 , the assumptions of the Crandall-Rabinowitz theorem are fulfilled.

Remark 3.5. The bifurcation points corresponding to $k=1$ are not the same for the Whitham and the $\mathrm{KdV}$ equation: we have $\mu_{1} \approx 0.87$ but $\mu^{*} \approx 0.83$.

Remark 3.6. In the original variables, before the transformation (3.6), the wave speed $\mu$ of the $\mathrm{KdV}$ equation becomes negative for $k \geq 3$, a fact related to that $\mathrm{KdV}$ is a proper model for small water waves when the wave speed is positive and the wave number small.

\section{Global bifurcation for the Whitham equation}

As earlier, let $\alpha>\frac{1}{2}$ denote a fixed Hölder exponent. Let $\mathrm{F}_{\mathrm{W}}$ be the Whitham operator from Theorem 3.1, defined by (3.2) and (3.4). With

$$
U:=\left\{(\varphi, \mu) \in C_{\text {even }}^{\alpha}(\mathbb{S}) \times(0,1): \varphi<\mu / 2\right\},
$$

we let

$$
S:=\left\{(\varphi, \mu) \in U: \mathrm{F}_{\mathrm{W}}(\varphi, \mu)=0\right\}
$$

be our set of solutions. By $\mathcal{L}(X)$ we denote the Banach algebra of bounded linear operators on a Banach space $X$, and by $\mathcal{L}_{\text {is }}(X)$ those which also have an inverse in $\mathcal{L}(X)$, i.e., those which are Banach space isomorphisms.

Lemma 4.1 ( $L^{\infty}$-bound). Let $\mu>0$. Any bounded Whitham solution satisfies

$$
\|\varphi\|_{\infty} \leq \mu+\left\|\mathrm{L}_{W}\right\|_{\mathcal{L}\left(L^{\infty}(\mathbb{S})\right)}
$$

Proof. From $\mu \varphi-\mathrm{L}_{\mathrm{W}} \varphi-\varphi^{2}=0$ we obtain that

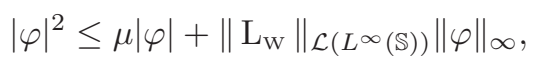

where we have used the fact that the Whitham kernel is integrable. Either $\varphi \equiv 0$, or we may take the supremum and divide by $\|\varphi\|_{\infty}$. In either case, (4.2) holds.

Lemma 4.2 (Fredholm). The Frechét derivative $\mathrm{D}_{\varphi} \mathrm{F}_{\mathrm{W}}[\varphi, \mu]$ is a Fredholm operator of index 0 for all $(\varphi, \mu) \in U$.

Proof. We have

$$
\mathrm{D}_{\varphi} \mathrm{F}_{\mathrm{W}}[\varphi, \mu]=(\mu-2 \varphi) \text { id }-\mathrm{L}_{\mathrm{W}},
$$

and, for any given $(\varphi, \mu) \in U$, that $(\mu-2 \varphi)$ id $\in \mathcal{L}_{\text {is }}\left(C_{\text {even }}^{\alpha}(\mathbb{S})\right)$. In view of that $\mathrm{L}_{\mathrm{W}}$ is compact on $C^{\alpha}(\mathbb{S})$, the operator $\mathrm{D}_{\varphi} \mathrm{F}_{\mathrm{W}}[\varphi, \mu]$ is Fredholm. The linearization $\mathrm{D}_{\varphi} \mathrm{F}_{\mathrm{W}}[0, \mu]$ has Fredholm index zero along the trivial solution curve; we have

$$
\tau \mapsto(\mu-2 \tau \varphi) \mathrm{id}-\mathrm{L}_{\mathrm{W}} \in C\left([0,1], \mathcal{L}\left(C^{\alpha}(\mathbb{S})\right)\right),
$$

and since the index is continuous in the operator-norm topology,it follows that it is zero also at $(\varphi, \mu)$. 
Lemma 4.3. Whenever $(\varphi, \mu) \in S$ the function $\varphi$ is smooth, and bounded and closed sets of $S$ are compact in $C_{\text {even }}^{\alpha}(\mathbb{S}) \times(0,1)$.

Proof. We write the Whitham equation in the form

$$
\varphi=\tilde{\mathrm{F}}(\varphi, \mu):=\frac{\mu}{2}-\left(\frac{\mu^{2}}{4}-\mathrm{L}_{\mathrm{w}} \varphi\right)^{1 / 2} .
$$

The mapping $\mathrm{L}_{\mathrm{W}}$ is bounded and linear $C^{\alpha}(\mathbb{S}) \rightarrow C^{\alpha+1 / 2}(\mathbb{S})$ (cf. Lemma 2.2), and $x \mapsto \sqrt{x}$ is real analytic for $x>0$. Consequently, if we let

$$
V:=\left\{(\varphi, \mu) \in C^{\alpha}(\mathbb{S}) \times(0,1): \frac{\mu^{2}}{4}>\mathrm{L}_{\mathrm{w}} \varphi\right\}
$$

then $\tilde{\mathrm{F}}$ is real analytic $V \rightarrow C^{\alpha+1 / 2}(\mathbb{S})$. The space $C^{\alpha+1 / 2}(\mathbb{S})$ is relatively compact in $C^{\alpha}(\mathbb{S})$, whence $\tilde{\mathrm{F}}$ maps bounded subsets of $V$ into pre-compact sets. We may then prove:

Smoothness. For any $\varphi \in S$ there exists a constant $R_{1}$ such that $\sup \varphi \leq R_{1}<\mu / 2$. Since $\varphi$ is a fixed point of $\tilde{\mathrm{F}}(\cdot, \mu)$ we have $(\varphi, \mu) \in V$. A straightforward induction argument reveals that $\varphi \in C^{\infty}(\mathbb{S})$.

Compactness. Let $K \subset S$ be bounded and closed in the $C^{\alpha}(\mathbb{S}) \times \mathbb{R}$-topology. Then $K \subset V$, and $\{\varphi:(\varphi, \mu) \in K\}=\tilde{\mathrm{F}} K$ is pre-compact in $C^{\alpha}(\mathbb{S})$. Any sequence $\left\{\left(\varphi_{j}, \mu_{j}\right)\right\}_{j>1} \subset K$ thus converges to a pair $\left(\varphi_{0}, \mu_{0}\right)$ in the $C^{\alpha}(\mathbb{S}) \times \mathbb{R}$-topology. The fact that $K$ is closed implies that $\left(\varphi_{0}, \mu_{0}\right) \in K$, whence $K$ is compact.

\subsection{Global bifurcation}

Using Lemmata 4.2 and 4.3 we shall now show that the local branches of Whitham solutions in Corollary 3.2 can be globally extended. The following result is immediate (cf. [6]) if we are able to show that, in some small neighborhood $|\varepsilon|<\delta, \mu(\varepsilon)$ is not identically equal to a constant.

Theorem 4.4 (Global bifurcation). The local bifurcation curves $\varepsilon \mapsto(\varphi(\varepsilon), \mu(\varepsilon))$ of solutions to the Whitham equation from Corollary 3.2 extend to global continuous curves of solutions $\mathbb{R}_{\geq 0} \rightarrow S$, with $S$ as in (4.1). One of the following alternatives holds:

(i) $\|\varphi(\varepsilon)\|_{C^{\alpha}(\mathbb{S})}$ is unbounded as $\varepsilon \rightarrow \infty$.

(ii) The pair $(\varphi(\varepsilon), \mu(\varepsilon))$ approaches the boundary of $S$ as $\varepsilon \rightarrow \infty$.

(iii) The function $\varepsilon \mapsto(\varphi(\varepsilon), \mu(\varepsilon))$ is $T$-periodic, for some $T \in(0, \infty)$.

Proof. According to [6, Thm 9.1.1], and in view of Lemmata 4.2 and 4.3, the assertion follows if any of the derivatives $\mu^{(k)}(0) \neq 0$. It will be shown in Theorem 4.6 that $\ddot{\mu}(0) \neq 0$.

In order to establish the bifurcation formulas for Whitham equation - and thereby Theorem 4.4-we apply the Lyapunov-Schmidt reduction (cf. [14]). Let $\mu^{*}:=\mu_{1}$ be the bifurcation point from Corollary 3.2 and let

$$
\varphi^{*}(x):=\cos (x) \text {. }
$$

Let furthermore

$$
M:=\left\{\sum_{k \neq 1} a_{k} \cos (k x) \in C^{\alpha}(\mathbb{S})\right\},
$$

and

$$
N:=\operatorname{ker}\left(\mathrm{D}_{\varphi} \mathrm{F}_{\mathrm{W}}\left[0, \mu^{*}\right]\right)=\operatorname{span}\left(\varphi^{*}\right)
$$


Then $C_{\text {even }}^{\alpha}(\mathbb{S})=M \oplus N$ and we can use the canonical embedding $C^{\alpha}(\mathbb{S}) \hookrightarrow L^{2}(\mathbb{S})$ to define a continuous projection

$$
\Pi \varphi:=\left\langle\varphi, \varphi^{*}\right\rangle_{L^{2}(\mathbb{S})} \varphi^{*}
$$

with $\langle u, v\rangle_{L^{2}(\mathbb{S})}:=\frac{1}{\pi} \int_{\mathbb{S}} u v d x$.

Theorem 4.5 (Lyapunov-Schmidt, [14]). There exists a neighborhood $\mathcal{O} \times Y \subset U$ around $\left(0, \mu^{*}\right)$ in which the problem

$$
\mathrm{F}_{W}(\varphi, \mu)=0
$$

is equivalent to that

$$
\Phi\left(\varepsilon \varphi^{*}, \mu\right):=\Pi \mathrm{F}_{W}\left(\varepsilon \varphi^{*}+\psi\left(\varepsilon \varphi^{*}, \mu\right), \mu\right)=0
$$

for functions $\psi \in C^{\infty}\left(\mathcal{O}_{N} \times Y, M\right), \Phi \in C^{\infty}\left(\mathcal{O}_{N} \times Y, N\right)$, and $\mathcal{O}_{N} \subset N$ an open neighborhood of the zero function in $N$. One has $\Phi\left(0, \mu^{*}\right)=0, \psi\left(0, \mu^{*}\right)=0, \mathrm{D}_{\varphi} \psi\left(0, \mu^{*}\right)=0$, and solving the finite-dimensional problem (4.5) provides a solution $\varphi=\varepsilon \varphi^{*}+\psi\left(\varepsilon \varphi^{*}, \mu\right)$ of the infinite-dimensional problem (4.4).

Theorem 4.6 (Bifurcation formulas). Let $\mu^{*}=\sqrt{\tanh (1)}, C_{1}:=\left(\mu^{*}-1\right)^{-1}$, and $C_{2}:=\left(2 \mu^{*}-\right.$ $\sqrt{2 \tanh (2)})^{-1}$. The main bifurcation curve $(k=1)$ for the Whitham equation found in Corollary 3.2 satisfies

$$
\varphi(\varepsilon)=\varepsilon \cos (x)+\varepsilon^{2}\left(\frac{1}{2} C_{1}+C_{2} \cos (2 x)\right)+\mathcal{O}\left(\varepsilon^{3}\right),
$$

and

$$
\mu(\varepsilon)=\mu^{*}+\varepsilon^{2}\left(C_{1}+C_{2}\right)+\mathcal{O}\left(\varepsilon^{3}\right),
$$

in $C_{\mathrm{even}}^{\alpha}(\mathbb{S}) \times(0,1)$ as $\varepsilon \rightarrow 0$. In particular, $\ddot{\mu}(0)<0$, Corollary 3.2 (i) describes a subcritical pitchfork bifurcation, and Theorem 4.4 holds.

Remark 4.7. Relying on Corollary 3.4 it is possible to calculate the asymptotic expansion

$$
\varphi_{\mathrm{KdV}}(\varepsilon)=\varepsilon \cos (x)+\varepsilon^{2}(\cos (2 x)-3)+\mathcal{O}\left(\varepsilon^{3}\right),
$$

in $C_{\text {even }}^{\alpha}(\mathbb{S})$ as $\varepsilon \rightarrow 0$ for the periodic $\mathrm{KdV}$-solutions found there. Although this agrees with (4.6) to the first order in $\varepsilon$, it does not for $\varepsilon^{2}$.

Proof. We perform first the analysis for $\mu$. We already know that $\varepsilon \mapsto \mu(\varepsilon)$ is analytic at $\varepsilon=0$ and that $\mu(0)=\mu^{*}$, so it remains to show that $\dot{\mu}(0)=0$ and to determine $\ddot{\mu}(0)$. We have

$$
\begin{aligned}
\mathrm{D}_{\varphi \varphi}^{2} \mathrm{~F}_{\mathrm{W}}\left[0, \mu^{*}\right]\left(\varphi^{*}, \varphi^{*}\right) & =-2 \varphi^{* 2}, \\
\mathrm{D}_{\varphi \mu}^{2} \mathrm{~F}_{\mathrm{W}}\left[0, \mu^{*}\right] \varphi^{*} & =\varphi^{*},
\end{aligned}
$$

and the value of $\dot{\mu}(0)$ may be explicitly calculated - for the bifurcation formulas used in this proof, we refer to $[14$, Section 1.6$]$-as

$$
\dot{\mu}(0)=-\frac{1}{2} \frac{\left\langle\mathrm{D}_{\varphi \varphi}^{2} \mathrm{~F}_{\mathrm{W}}\left[0, \mu^{*}\right]\left(\varphi^{*}, \varphi^{*}\right), \varphi^{*}\right\rangle_{L^{2}(\mathbb{S})}}{\left\langle\mathrm{D}_{\varphi \mu}^{2} \mathrm{~F}_{\mathrm{W}}\left[0, \mu^{*}\right] \varphi^{*}, \varphi^{*}\right\rangle_{L^{2}(\mathbb{S})}}=0,
$$

since $\int_{\mathbb{S}} \cos ^{3}(x) d x=0$.

Moreover, when $\dot{\mu}(0)=0$ one has that

$$
\ddot{\mu}(0)=-\frac{1}{3} \frac{\left\langle\mathrm{D}_{\varphi \varphi \varphi}^{3} \Phi\left[0, \mu^{*}\right]\left(\varphi^{*}, \varphi^{*}, \varphi^{*}\right), \varphi^{*}\right\rangle_{L^{2}(\mathbb{S})}}{\left\langle\mathrm{D}_{\varphi \mu}^{2} \mathrm{~F}_{\mathrm{W}}\left[0, \mu^{*}\right] \varphi^{*}, \varphi^{*}\right\rangle_{L^{2}(\mathbb{S})}} .
$$


Since $\mathrm{D}_{\varphi \mu}^{2} \mathrm{~F}_{\mathrm{w}}\left[0, \mu^{*}\right]=\mathrm{id}$ we find that the denominator is of unit size. One calculates

$$
\begin{aligned}
& \mathrm{D}_{\varphi} \Phi[\varphi, \mu] \varphi^{*}=\Pi \mathrm{D}_{\varphi} \mathrm{F}_{\mathrm{W}}[\varphi+\psi(\varphi, \mu), \mu]\left(\varphi^{*}+\mathrm{D}_{\varphi} \psi(\varphi, \mu) \varphi^{*}\right), \\
& \mathrm{D}_{\varphi \varphi}^{2} \Phi[\varphi, \mu]\left(\varphi^{*}, \varphi^{*}\right) \\
& =\Pi \mathrm{D}_{\varphi \varphi}^{2} \mathrm{~F}_{\mathrm{W}}[\varphi+\psi(\varphi, \mu), \mu]\left(\varphi^{*}+\mathrm{D}_{\varphi} \psi[\varphi, \mu] \varphi^{*}, \varphi^{*}+\mathrm{D}_{\varphi} \psi[\varphi, \mu] \varphi^{*}\right) \\
& \quad+\Pi \mathrm{D}_{\varphi} \mathrm{F}_{\mathrm{W}}[\varphi+\psi(\varphi, \mu), \mu] \mathrm{D}_{\varphi \varphi}^{2} \psi[\varphi, \mu]\left(\varphi^{*}, \varphi^{*}\right),
\end{aligned}
$$

and, in view of that $\mathrm{F}_{\mathrm{W}}$ is quadratic in $\varphi$,

$$
\begin{aligned}
& \mathrm{D}_{\varphi \varphi \varphi}^{3} \Phi[\varphi, \mu]\left(\varphi^{*}, \varphi^{*}, \varphi^{*}\right) \\
& =3 \Pi \mathrm{D}_{\varphi \varphi}^{2} \mathrm{~F}_{\mathrm{W}}[\varphi+\psi(\varphi, \mu), \mu]\left(\varphi^{*}+\mathrm{D}_{\varphi} \psi[\varphi, \mu] \varphi^{*}, \mathrm{D}_{\varphi \varphi}^{2} \psi[\varphi, \mu]\left(\varphi^{*}, \varphi^{*}\right)\right) \\
& \quad+\Pi \mathrm{D}_{\varphi} \mathrm{F}_{\mathrm{W}}[\varphi+\psi(\varphi, \mu), \mu] \mathrm{D}_{\varphi \varphi \varphi}^{3} \psi[\varphi, \mu]\left(\varphi^{*}, \varphi^{*}, \varphi^{*}\right) .
\end{aligned}
$$

Using the form of $\mathrm{D}_{\varphi} \mathrm{F}_{\mathrm{W}}$ together with that $\psi\left(0, \mu^{*}\right)=\mathrm{D}_{\varphi} \psi\left[0, \mu^{*}\right] \varphi^{*}=0$ one finds that

$$
\begin{aligned}
\mathrm{D}_{\varphi \varphi \varphi}^{3} \Phi\left[0, \mu^{*}\right]\left(\varphi^{*}, \varphi^{*}, \varphi^{*}\right)= & \Pi\left(\mu^{*} \mathrm{id}-\mathrm{L}_{\mathrm{W}}\right) \mathrm{D}_{\varphi \varphi \varphi}^{3} \psi\left[0, \mu^{*}\right]\left(\varphi^{*}, \varphi^{*}, \varphi^{*}\right) \\
& -6 \Pi \varphi^{*} \mathrm{D}_{\varphi \varphi}^{2} \psi\left[0, \mu^{*}\right]\left(\varphi^{*}, \varphi^{*}\right) .
\end{aligned}
$$

We have $\operatorname{ran}\left(\mu^{*} \mathrm{id}-\mathrm{L}_{\mathrm{W}}\right)=M$, so that $\Pi\left(\mu^{*}\right.$ id $\left.-\mathrm{L}_{\mathrm{W}}\right)=0$. We thus need to determine $\varphi^{*} \mathrm{D}_{\varphi \varphi}^{2} \psi\left[0, \mu^{*}\right]\left(\varphi^{*}, \varphi^{*}\right)$. Since $\mathrm{D}_{\varphi} \mathrm{F}_{\mathrm{W}}\left[0, \mu^{*}\right]=\mu^{*} \mathrm{id}-\mathrm{L}_{\mathrm{W}}$ is an isomorphism on $M$, it is possible (see again $\left[14\right.$, Section 1.6]) to rewrite $\mathrm{D}_{\varphi \varphi}^{2} \psi\left[0, \mu^{*}\right]\left(\varphi^{*}, \varphi^{*}\right)$ as

$$
\begin{aligned}
& \mathrm{D}_{\varphi \varphi}^{2} \psi\left[0, \mu^{*}\right]\left(\varphi^{*}, \varphi^{*}\right) \\
& =-\left(\mathrm{D}_{\varphi} \mathrm{F}_{\mathrm{W}}\left[0, \mu^{*}\right]\right)^{-1}(\mathrm{id}-\Pi) \mathrm{D}_{\varphi \varphi} \mathrm{F}_{\mathrm{W}}\left[0, \mu^{*}\right]\left(\varphi^{*}, \varphi^{*}\right) \\
& =-\left(\mathrm{D}_{\varphi} \mathrm{F}_{\mathrm{W}}\left[0, \mu^{*}\right]\right)^{-1}(\mathrm{id}-\Pi)\left(-2 \varphi^{* 2}\right) \\
& =\left(\mathrm{D}_{\varphi} \mathrm{F}_{\mathrm{W}}\left[0, \mu^{*}\right]\right)^{-1}(1+\cos (2 x)) \\
& =\frac{1}{\mu^{*}-1}+\frac{\cos (2 x)}{\mu^{*}-\sqrt{\tanh (2) / 2}}
\end{aligned}
$$

After multiplication with $\cos (x)$ this equals

$$
\frac{\cos (x)}{\mu^{*}-1}+\frac{\cos (x)}{2\left(\mu^{*}-\sqrt{\tanh (2) / 2}\right)}+\frac{\cos (3 x)}{2\left(\mu^{*}-\sqrt{\tanh (2) / 2}\right)} .
$$

In view of (4.8) and (4.9) the coefficient in front of $\cos x$ equals $\frac{1}{2} \ddot{\mu}(0)$. All taken into consideration, we obtain (4.7) via a Maclaurin series, and one easily checks that $\ddot{\mu}(0)<0$.

To prove (4.6) one makes use of the formula

$$
\varphi(\varepsilon)=\varepsilon \varphi^{*}+\psi\left(\varepsilon \varphi^{*}, \mu(\varepsilon)\right)
$$

from the Lyapunov-Schmidt reduction (cf. Theorem 4.5). We already know that $\varphi(0)=0$ and $\dot{\varphi}(0)=$ $\cos (x)$, so it remains to calculate $\ddot{\varphi}(0)$. It follows from (4.11) that

$$
\begin{aligned}
\ddot{\varphi}(\varepsilon)= & \mathrm{D}_{\varphi \varphi}^{2} \psi\left[0, \mu^{*}\right]\left(\varphi^{*}, \varphi^{*}\right)+2 \mathrm{D}_{\varphi \mu}^{2} \psi\left[0, \mu^{*}\right]\left(\varphi^{*}, \dot{\mu}(0)\right) \\
& +\mathrm{D}_{\mu \mu}^{2} \psi\left[0, \mu^{*}\right](\dot{\mu}(0), \dot{\mu}(0))+\mathrm{D}_{\mu} \psi\left[0, \mu^{*}\right] \dot{\mu}(0) .
\end{aligned}
$$

Since $\psi(0, \mu) \equiv 0$ where $\psi$ exists, we have $\mathrm{D}_{\mu} \psi\left(0, \mu^{*}\right)=0$. Combining this with $\dot{\mu}(0)=0$ one finds that

$$
\ddot{\varphi}(0)=\mathrm{D}_{\varphi \varphi}^{2} \psi\left[0, \mu^{*}\right](\cos (x), \cos (x)),
$$

so that the proposition now follows from (4.10). 


\subsection{Properties along the bifurcation branch}

Theorem 4.8 (Uniform convergence). Any sequence of Whitham solutions $\left(\varphi_{n}, \mu_{n}\right) \in S$ has a subsequence which converges uniformly to a solution $\varphi \in C^{\alpha}(\mathbb{S})$, with $\alpha \in\left(0, \frac{1}{2}\right)$ arbitrary. At any point where $\varphi<\frac{\mu}{2}$ the solution is $\alpha$-Hölder continuous for all $\alpha \in(0,1)$.

Proof. Since $\mu_{n} \in(0,1)$, Lemma 4.1 implies that $\varphi_{n}$ is uniformly bounded in $C(\mathbb{S})$. The proof of Theorem 4.1 in [10] shows that any uniformly bounded sequence of Whitham solutions (i.e. in $L_{\infty}(\mathbb{R})$ ) is equicontinuous; this follows from a standard argument using the integral kernel of $\mathrm{L}_{\mathrm{W}}$. Since we are dealing with periodic solutions, we may apply the Arzola-Ascoli lemma to conclude that a subsequence of $\varphi_{n}$ converges in $C(\mathbb{S})$.

Since $\mathrm{L}_{\mathrm{W}}$ maps $C(\mathbb{S})$ into $C^{1 / 2}(\mathbb{S})$ (cf. Lemma 2.2 ) we see from $(4.3)$ that $\varphi \in C^{1 / 2}(\mathbb{S})$ wherever $2 \varphi \neq \mu$. On the other hand, when $\varphi(x)=\frac{\mu}{2}$ we have that

$$
\begin{aligned}
|\varphi(x)-\varphi(y)| & =\frac{\mu}{2}-\varphi(y)=\left(\frac{\mu^{2}}{4}-\mathrm{L}_{\mathrm{W}} \varphi(y)\right)^{1 / 2} \\
& =\left(\mathrm{L}_{\mathrm{W}} \varphi(x)-\mathrm{L}_{\mathrm{W}} \varphi(y)\right)^{1 / 2} \leq C|x-y|^{1 / 4}
\end{aligned}
$$

in view of that $\mathrm{L}_{\mathrm{W}} \varphi$ is $\alpha$-Hölder continuous for $\alpha=\frac{1}{2}$. Hence $\varphi \in C^{1 / 4}(\mathbb{S})$. Repeating this argument once shows that $\varphi \in C^{3 / 4}$ wherever $2 \varphi \neq \mu$, and $\varphi \in C^{3 / 8}(\mathbb{S})$. Bootstrapping yields Hölder continuity below $\alpha=1$ for $2 \varphi \neq \mu$, and below $\alpha=\frac{1}{2}$ for $\varphi \in C^{\alpha}(\mathbb{S})$.

Proposition 4.9 (Characterization of blow-up). Alternative (i) in Theorem 4.4 can happen only if

$$
\liminf _{\varepsilon \rightarrow \infty} \inf _{x \in \mathbb{R}}\left(\frac{\mu(\varepsilon)}{2}-\varphi(x ; \varepsilon)\right)=0 .
$$

In particular, alternative (i) implies alternative (ii).

Proof. Assume that $\liminf _{\varepsilon \rightarrow \infty} \inf _{x}\left(\frac{\mu(\varepsilon)}{2}-\varphi(x ; \varepsilon)\right) \geq \delta$, for some $\delta>0$. Any such solution of the Whitham equation satisfies

$$
|\varphi(x)-\varphi(y)|=\frac{\left|\mathrm{L}_{\mathrm{W}} \varphi(x)-\mathrm{L}_{\mathrm{W}} \varphi(y)\right|}{\mu-\varphi(x)-\varphi(y)} \leq \frac{\left|\mathrm{L}_{\mathrm{W}} \varphi(x)-\mathrm{L}_{\mathrm{W}} \varphi(y)\right|}{2 \delta} .
$$

Since $\mathrm{L}_{\mathrm{W}}$ is continuous $C(\mathbb{S}) \rightarrow C^{1 / 2}(\mathbb{S})$ and the family $\{\varphi(\varepsilon)\}_{\varepsilon}$ is uniformly bounded in $C(\mathbb{S})$ (cf. Lemma 4.1), it follows that $\{\varphi(\varepsilon)\}_{\varepsilon}$ is uniformly bounded in $C^{1 / 2}(\mathbb{S})$ too. Repeating the argument for $\mathrm{L}_{\mathrm{W}}$ as a continuous operator $C^{1 / 2}(\mathbb{S}) \rightarrow C^{\alpha}(\mathbb{S}), \alpha<1$, yields that

$$
\|\varphi(\varepsilon)\|_{C^{\alpha}(\mathbb{S})} \leq C \delta^{-2}, \quad \alpha \in(0,1)
$$

for some constant $C$ depending only $\mathrm{L}_{\mathrm{w}}$ (recall that $\mu$ is bounded by assumption). This shows that $\|\varphi(\varepsilon)\|_{C^{\alpha}(\mathbb{S})} \rightarrow \infty$ is possible only if (4.12) holds.

\subsection{Summary}

In Section 4 we have shown that each of the subcritical bifurcation branches in Corollary 3.2 (i) can be extended to a global bifurcation branch in the set $S$ of solution pairs $(\varphi, \mu) \in C_{\text {even }}^{\alpha}(\mathbb{S}) \times(0,1)$ satisfying $\varphi<\mu / 2$. These solutions are all smooth. Each bifurcation curve contains a sequence of solutions which converge in $C^{0}$ to a solution of Hölder regularity $\alpha \in\left(0, \frac{1}{2}\right)$, with the better pointwise regularity $\alpha \in(0,1)$ at any point $x$ where $\varphi(x)<\mu / 2$. If the $C^{\alpha}$-norm, $\alpha>\frac{1}{2}$, blows up along the bifurcation branch, the corresponding sequence of solutions approaches $\varphi=\frac{\mu}{2}$ in the sense of (4.12). In spite of this - and in spite of the numerical evidence available - an analytical proof of a 'highest', cusped, limiting wave at the end of each bifurcation branch remains an open problem. 


\section{Spectral schemes for the Whitham equation}

In this section, the numerical approximation of solutions of (1.1) is in view. Both for the approximation of traveling waves and for the discretization of the evolution problem, spectral schemes are used. This is a natural choice since the operator defining the linear part of the equations is given in terms of Fourier multipliers.

\subsection{Traveling waves}

Solutions of (2.1) are approximated using a spectral cosine collocation method. To define the cosinecollocation projection, first define the subspace

$$
S_{N}=\operatorname{span}_{\mathbb{R}}\{\cos (l x) \mid 0 \leq l \leq N-1\}
$$

of $L^{2}(0, \pi)$, and the collocation points $x_{n}=\pi \frac{2 n-1}{N}$ for $n=1, \ldots, N$. The discretization is defined by seeking $\phi_{N}$ in $S_{N}$ satisfying the equation

$$
-\mu \phi_{N}+\phi_{N}^{2}+K^{N} \phi_{N}=0
$$

where the operator $K^{N}$ the discrete form of $K$ defined in (2.2). The discrete cosine representation of $\phi_{N}$ given by

$$
\phi_{N}(x)=\sum_{l=0}^{N-1} w(l) \Phi_{N}(l) \cos (l x)
$$

where

$$
w(l)= \begin{cases}\sqrt{1 / N}, & l=0 \\ \sqrt{2 / N}, & l \geq 1\end{cases}
$$

and $\Phi_{N}(l)$ are the discrete cosine coefficients, given by

$$
\Phi_{N}(l)=w(l) \sum_{n=1}^{N} \phi_{N}\left(x_{n}\right) \cos \left(l x_{n}\right), \quad \text { for } l=0, \ldots, N-1 .
$$

Now if the equation (5.1) is enforced at the collocation points $x_{n}$, the term $K^{N} \phi_{N}$ may be practically evaluated with the help of the matrix $\left[K^{N}\right](m, n)$ by

$$
\left[K^{N}\right] \phi_{N}\left(x_{m}\right)=\sum_{n=1}^{N}\left[K^{N}\right](m, n) \phi_{N}\left(x_{n}\right),
$$

where the matrix $\left[K^{N}\right](m, n)$ is defined by

$$
\left[K^{N}\right](m, n)=\sum_{l=0}^{N-1} w^{2}(l) \sqrt{\frac{1}{l} \tanh l} \cos \left(l x_{n}\right) \cos \left(l x_{m}\right) .
$$

Thus, equation (5.1) enforced at the collocation points $x_{n}$ yields a system of $N$ nonlinear equations, which can be efficiently solved using a Newton method. The cosine expansion effectively removes the singularities of the Jacobian matrix due to translational invariance and symmetry of the solutions. The nondimensional speed $\mu$ is used as the bifurcation parameter, but as the turning point is approached, it is more convenient to change to a waveheight parametrization. The computation can be started for $\mu$ close to but smaller than the critical speed $\mu_{k}$, and with an initial guess for the first computation given by comparing (4.6) and (4.7).

In Figure 1, a periodic traveling wave with speed $\mu=0.789$ is shown. In panel (a), the function is drawn on the interval $[0,2 \pi]$, and the collocation values are indicated with dots in the curve. In panel 

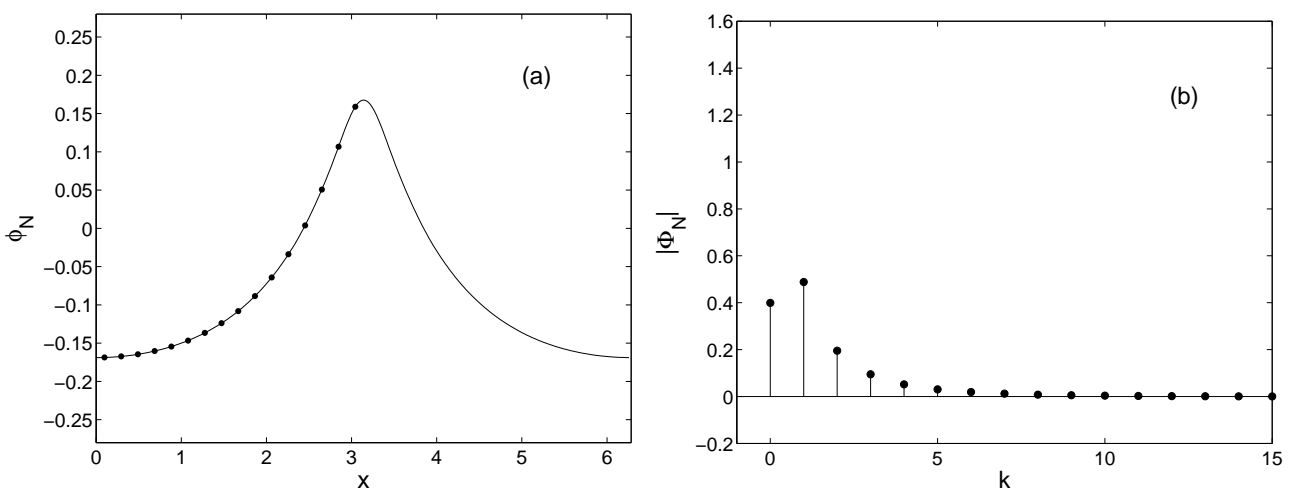

FiguRE 1. An approximate traveling-wave solution $\phi_{N}$ with nondimensional wavespeed $\mu=0.789$, wavelength $2 \pi$, and nondimensional waveheight 0.3368 is shown in the left panel. The collocation approximation used 16 gridpoints, which are indicated as dots in the graph. The amplitudes of the discrete Fourier modes are shown on the right.
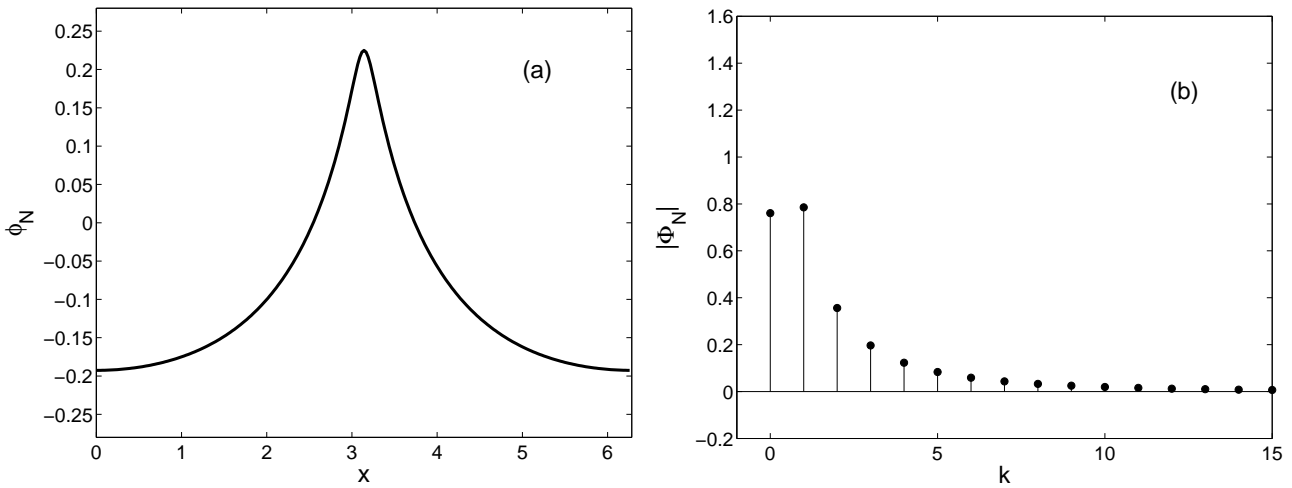

FigURE 2. An approximate traveling-wave solution $\phi_{N}$ with nondimensional wavespeed $\mu=0.7715$, wavelength $2 \pi$, and nondimensional waveheight 0.4152 . The collocation approximation used 64 gridpoints, and the amplitudes of the discrete Fourier modes are shown on the right.

(b), the values of the discrete cosine coefficients are shown. This computation was done with only 16 modes. In Figure 2, a periodic traveling wave with speed $\mu=0.7715$ is shown. It appears that the wave is steeper, and higher cosine coefficients are important. The computation was done with 64 modes. To make sure that the computed functions are approximate traveling waves for the Whitham equation, we have also used a dynamic integrator for the time-dependent Whitham equation, as shown in the next subsection.

\subsection{Time-dependent discretization}

For the purpose of approximating time-dependent periodic solutions of (1.1), a Fourier method is optimal. To define the Fourier-collocation projection, the subspace

$$
\mathcal{S}_{N}=\operatorname{span}_{\mathbb{C}}\{\exp (i k x) \mid k \in \mathbb{Z},-N / 2 \leq k \leq N / 2-1\}
$$

of $L^{2}(0,2 \pi)$ is used. The collocation points are defined to be $x_{j}=\frac{2 \pi j}{N}$ for $j=0,1, \ldots, N-1$. Note that $N$ here is in general different from the $N$ used in the previous section. Let $P_{N}$ be the projection operator 
onto $\mathcal{S}_{N}$, and let $I_{N}$ be the interpolation operator from $C_{p e r}^{\infty}([0,2 \pi])$ onto $\mathcal{S}_{N}$. As explained in $[3,7]$, this operator is defined for a given $u \in C_{p e r}^{\infty}([0,2 \pi])$, by letting $I_{N} u$ be the unique element of $\mathcal{S}_{N}$ that coincides with $u$ at the collocation points $x_{j}$. The same scaling as in the steady case is used, so that the non-dimensional form of the equation is

$$
\eta_{t}+2 \eta \eta_{x}+K * \eta_{x}=0
$$

The spatial discretization is then defined by the following problem. Find a function $\eta_{N}:[0, T] \rightarrow \mathcal{S}_{N}$, such that

$$
\left\{\begin{array}{l}
\partial_{t} \eta_{N}+\partial_{x} I_{N}\left(\eta_{N}^{2}\right)+\mathcal{K}^{N} * \partial_{x} \eta_{N}=0, x \in[0,2 \pi] \\
\eta_{N}(\cdot, 0)=P_{N} \eta_{0}
\end{array}\right.
$$

where $\eta_{0}$ is the initial data. If $\eta_{N}(\cdot, t)$ is written in terms of its discrete Fourier coefficients $\tilde{\eta}_{N}(k)$ as

$$
\eta_{N}(x)=\sum_{-N / 2 \leq k \leq N / 2-1} \tilde{\eta}_{N}(k) \exp (i k x)
$$

the operator $\mathcal{K}^{N}$ can be evaluated using the formula

$$
\mathcal{K}^{N} \eta_{N}(x)=\tilde{\eta}_{N}(0)+\sum_{\substack{1-N / 2 \leq k \leq N / 2-1 \\ k \neq 0}} \sqrt{\frac{1}{k} \tanh k} \tilde{\eta}_{N}(k) \exp (i k x) .
$$

Thus the operator $\mathcal{K}^{N}$ is the truncation at the $N / 2$-st Fourier mode of the operator given by the periodic convolution with $K$. Note that this formulation includes the truncation of the Fourier mode $\tilde{\phi}_{N}(-N / 2)$ which ensures that the solution stays real, and which otherwise can lead to instabilities in the computation. The time integration of (5.3) may be carried out in a number of ways. We have chosen to use a midpoint method for both the linear and the nonlinear term. This method can be motivated as follows. Suppose the discrete solution is known at the time $t$. Then by the fundamental theorem of calculus, it is clear that

$$
\eta_{N}\left(x_{j}, t+\delta t\right)=\eta_{N}\left(x_{j}, t\right)+\int_{t}^{t+\delta t} \partial_{t} \eta\left(x_{j}, s\right) d s .
$$

Now using the equation (5.4) and approximating the integral with help of the trapezoidal rule yields

$$
\begin{aligned}
\eta_{N}\left(x_{j}, t+\delta t\right)=\eta_{N}\left(x_{j}, t\right)+\frac{\delta t}{2}\left\{\partial_{x} I_{N}\left(\eta_{N}^{2}\left(x_{j}, t\right)+\eta_{N}^{2}\left(x_{j}, t+\delta t\right)\right)\right\} & \\
& +\frac{\delta t}{2}\left\{\mathcal{K}^{N} \eta_{N}\left(x_{j}, t\right)+\mathcal{K}^{N} \eta_{N}\left(x_{j}, t+\delta t\right)\right\}+\mathcal{O}\left(\delta t^{3}\right) .
\end{aligned}
$$

From this equation, one may easily construct a fully discrete approximation. This can be done either in physical space, or in Fourier space. Constructing the approximation in Fourier space appears to be more convenient for the time-dependent problem. Since nonlinear terms are involved, the method is in effect pseudo-spectral, that is, derivatives and integral operators are evaluated in Fourier space, while nonlinear terms are taken in physical variables. In practice, the term $\eta_{N}^{2}\left(x_{j}, t+\delta t\right)$ is approximated by $\eta_{N}^{2}\left(x_{j}, t\right)$, and the equation is solved for $\eta_{N}\left(x_{j}, t+\delta t\right)$. Then this is used as a new approximation for the square term. This procedure is iterated until the change in the result falls below a certain tolerance. This scheme is second-order in time, so that a relatively small time step has to be used. Of course, this also means that the number of iterations at each time step is small, typically less than three.

In Table 1, we record the numerical errors incurred by the time integration of an approximate traveling wave with velocity $\mu=0.8615$ propagating for 1 and 100 periods. To find the most advantageous combinations of the number of Fourier modes $N$ and the time step $\delta t$, we used a computation for one period. We then use this combination, and integrated for 100 periods. The discrete $L^{2}$-error, the difference in maximal height between the original wave, and the profile after 1 and 100 periods were computed. 
TABLE 1. Error in evolution code after 5 and 50 periods for the traveling wave shown in Figure 1.

\begin{tabular}{cccccc}
\hline $\mathrm{N}$ & $\delta t$ & $L^{2}$-error & $|u|_{\infty}-\left|u_{N}\right|_{\infty}$ & $L^{2}$-error & $|u|_{\infty}-\left|u_{N}\right|_{\infty}$ \\
\hline $2^{5}$ & $2^{-10}$ & $2.675 \mathrm{e}-04$ & $2.838 \mathrm{e}-05$ & $9.635 \mathrm{e}-04$ & $2.612 \mathrm{e}-05$ \\
$2^{6}$ & $2^{-14}$ & $2.644 \mathrm{e}-05$ & $1.876 \mathrm{e}-06$ & $5.299 \mathrm{e}-05$ & $1.587 \mathrm{e}-06$ \\
$2^{7}$ & $2^{-19}$ & $1.021 \mathrm{e}-06$ & $5.876 \mathrm{e}-08$ & $4.489 \mathrm{e}-07$ & $5.286 \mathrm{e}-08$ \\
\hline \multicolumn{7}{c}{1 period } & \multicolumn{2}{c}{100 periods } \\
\hline
\end{tabular}
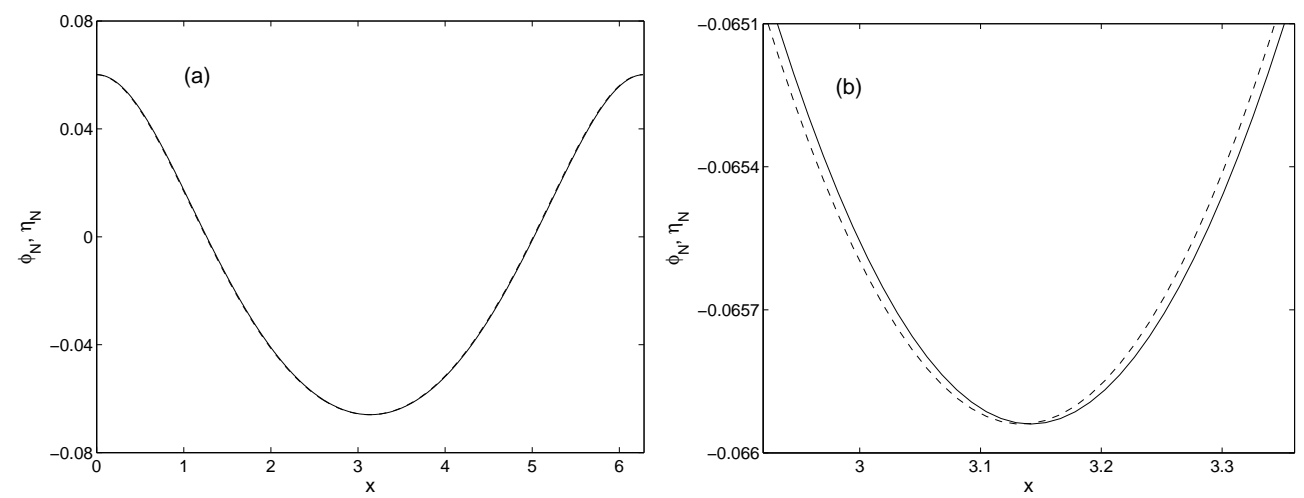

FiguRE 3. Solid line: approximate traveling wave $\phi_{N}$ for the Whitham equation $\mu=$ 0.8615. Dashed line: $\eta_{N}$ after time integration using (5.4) for 10000 periods. In (a), the difference between $\phi_{N}$ and $\eta_{N}$ is hardly visible. In this computation, $N=512$ and $\delta t=5 e-04$. The $L^{2}$ error was $2.1 e-03$, while the difference in height was $2.24 e-06$. This and the magnification (b) suggests that the the error is mainly due to a phase shift.

As can be seen, the error drops when increasing $N$ or when decreasing $h$. Moreover, the fact that the difference in maximal height is generally smaller than the $L^{2}$-error suggests that the error incurred during the time evolution is mostly due to a phase shift of the solution. This can also be observed in Figure 3 , where the same traveling wave is shown after time integration for 10000 periods. These results also suggest that the traveling waves are orbitally stable, but no special investigation of this question has been carried out.

\section{Numerical results}

In this section, we present a few numerical computations carried out using the discretizations described above. First, we will present an in-depth study of some bifurcation branches of the Whitham equation, and then the Whitham waves will be compared to traveling-wave solutions of the KdV equation.

\subsection{The Whitham branch}

In Figures 1 and 2, traveling wave solutions of the Whitham equation were shown. Figure 4 shows the bifurcation branch of these waves. In Figure 4(a), the whole branch is shown, and compared to the formula given in (4.7). In Figure 4(b) a close-up of the turning point is shown. Determining the endpoint of the branch is challenging. Here a combination of two tests are used. First, if a point is suspected to be higher than the highest branchpoint, then the solution is interpolated, and the Newton scheme run on a finer grid. Failure of this scheme to converge indicates that the point does not lie on the branch. The highest point in Figure 4(b) corresponds to a wave for which the Newton scheme converged with more than 1400 gridpoints on the interval $[0,2 \pi]$. The second test uses the dynamic integrator. For example, 

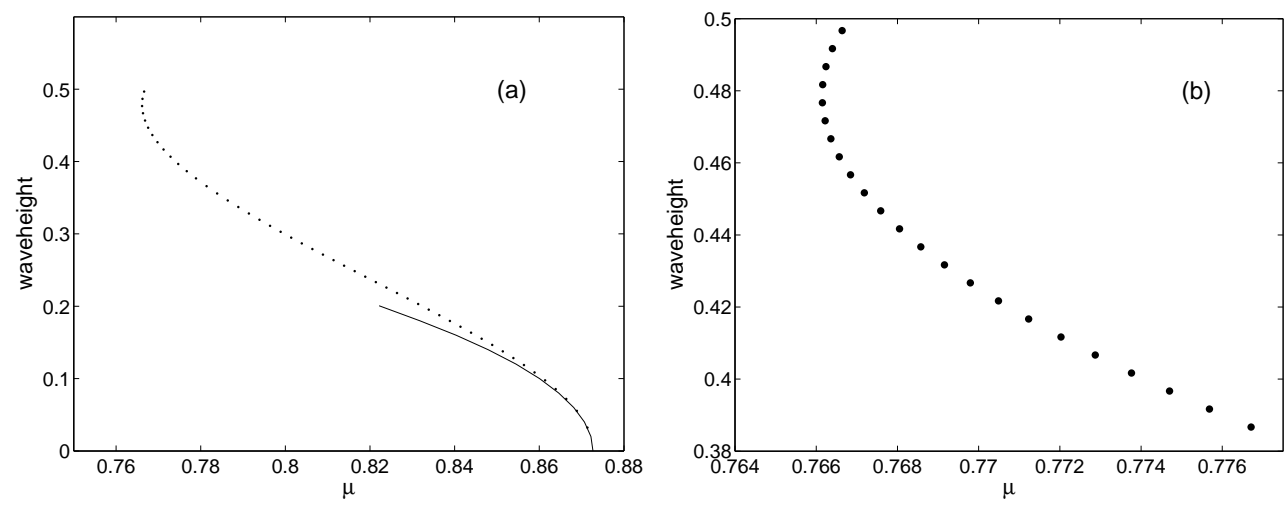

FiguRE 4. Bifurcation branch of $2 \pi$-periodic traveling wave solutions of the Whitham equation. In (a), the entire bifurcation branch is indicated with dots, and compared to a curve described by the expressions given in (4.7) and (4.6). In (b), a close-up of the turning point is shown.
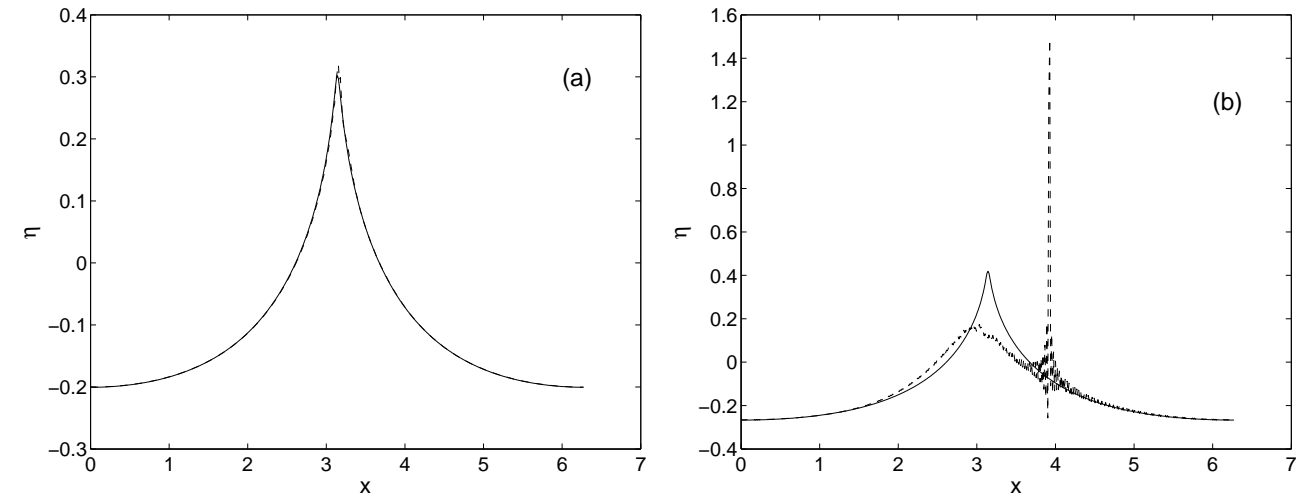

FiguRE 5. This figure presents computations using the dynamic discretization with approximate Whitham traveling waves as initial data. The computations were done with $N=256$ and time step $\delta t=0.001$. The initial data $\phi_{N}$ is represented by the solid curve, and the computed profile $\eta_{N}$ after one period is represented by the dashed curve. In (a), a wave with wavespeed $c=0.7664$ is shown, In (b), a wave with wavespeed $c=0.7667$ is shown.

Figure 5(a) shows the results of running the wave corresponding to the highest point on the branch shown in Figure 3 through the dynamic integrator for one period. Figure $5(\mathrm{~b})$ shows the next point (which is not shown in Figure 4), but which can be computed with lower values of $N$ (up to about $N=400$ ). The difference is dramatic, and the time integration cannot be rescued by choosing a smaller time step. Nevertheless, we do not expect the numerical scheme to be able to approximate the wave corresponding to the very highest point on the branch. Indeed, comparing the maximum of the highest wave shown in figures 4 and 6 to the statement of Theorem 4.8, it appears that the branch of Hölder continuous solutions should continue further than shown in those figures. On the other hand, our criterion for termination of the numerical bifurcation included failure of the time-dependent scheme to converge, and this failure can also be a result of instability of the computed traveling wave. Moreover, if the highest wave does indeed feature a cusp, then the spectral scheme will fail to converge, and convergence for waves close to 

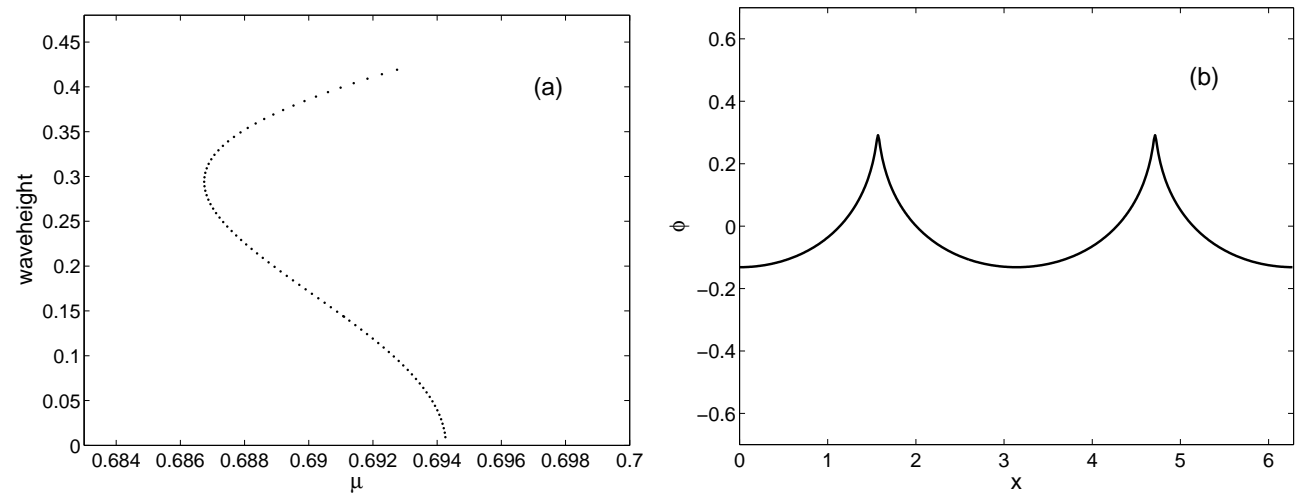

FiguRE 6. A branch of approximate traveling waves for the Whitham equation with $k=2$ is shown in (a). The profile of the highest wave on $[0,2 \pi]$ is shown in (b).

the terminal wave will also be delicate. A method for computing cusped waves was proposed in [4], and this might be a good approach for possible further studies.

In Figure 6, a branch with $k=2$ is shown. To find the highest point on this branch, similar methodology as for the principal branch was used. We note that the turning point is much farther from the end of the branch than in the principal branch.

\subsection{Comparison with $\mathrm{KdV}$}

It is of interest to see how the change in the linear dispersion relation affects the shape of the traveling waves. Therefore, attention will now be focused on the comparison of traveling waves of the Whitham equation to traveling-wave solutions of the KdV equation.

First the bifurcation branches are compared. For this purpose, the wavelength is held fixed at $2 \pi$. It was noted in Section 3 that the bifurcation points $\mu_{1}$ and $\mu^{*}$ are not the same. This difference is due to the small wavelength $2 \pi$. A comparison using a larger wavelength would yield a smaller difference in bifurcation points. Figure 7(a) shows bifurcation branches for both KdV (solid curve) and Whitham (dotted curve) equations. As noted above, the Whitham branch appears to have a highest wave, after which the approximation no longer converges. On the other hand, the KdV branch is unlimited.

For the purpose of comparing the shape of the waves, we chose to fix the waveheight and wavelength of the traveling waves. Other normalizations are of course possible, such as fixing the speed or some integral measure. In Figure 7(b), (c) and (d) Whitham and KdV traveling waves with respective waveheights of 0.1, 0.4 and 0.485 are shown. For a better comparison, the waves are plotted with the minimum at zero. The small-amplitude waves are very similar in shape. However, for the large-amplitude waves we note that the Whitham waves appear narrower, with a more pronounced and sharp peak.

\section{Conclusion}

In this article, a bifurcation theory has been developed to prove existence of a global branch of travelingwaves solutions of the Whitham equations (1.1). Smoothness of the solutions lying on the bifurcation curve has been proved, and it has been shown that the solutions converge uniformly to a solution of Hölder regularity $\alpha \in(0,1)$, expect possibly at the highest crest point (where $\alpha$ may be less than $1 / 2$ ). The larger Hölder norm blows up only if the highest point is attained. It was conjectured already by Whitham [19] that this branch of traveling wave has a limiting wave which has a cusp. Unfortunately such a result has not been established, but remains an interesting open problem. A comparative local bifurcation was provided for the Whitham and the KdV equations, and numerical tools were used to exhibit properties of the principal branch $(k=1)$ and the secondary bifurcation branch $(k=2)$ of the 

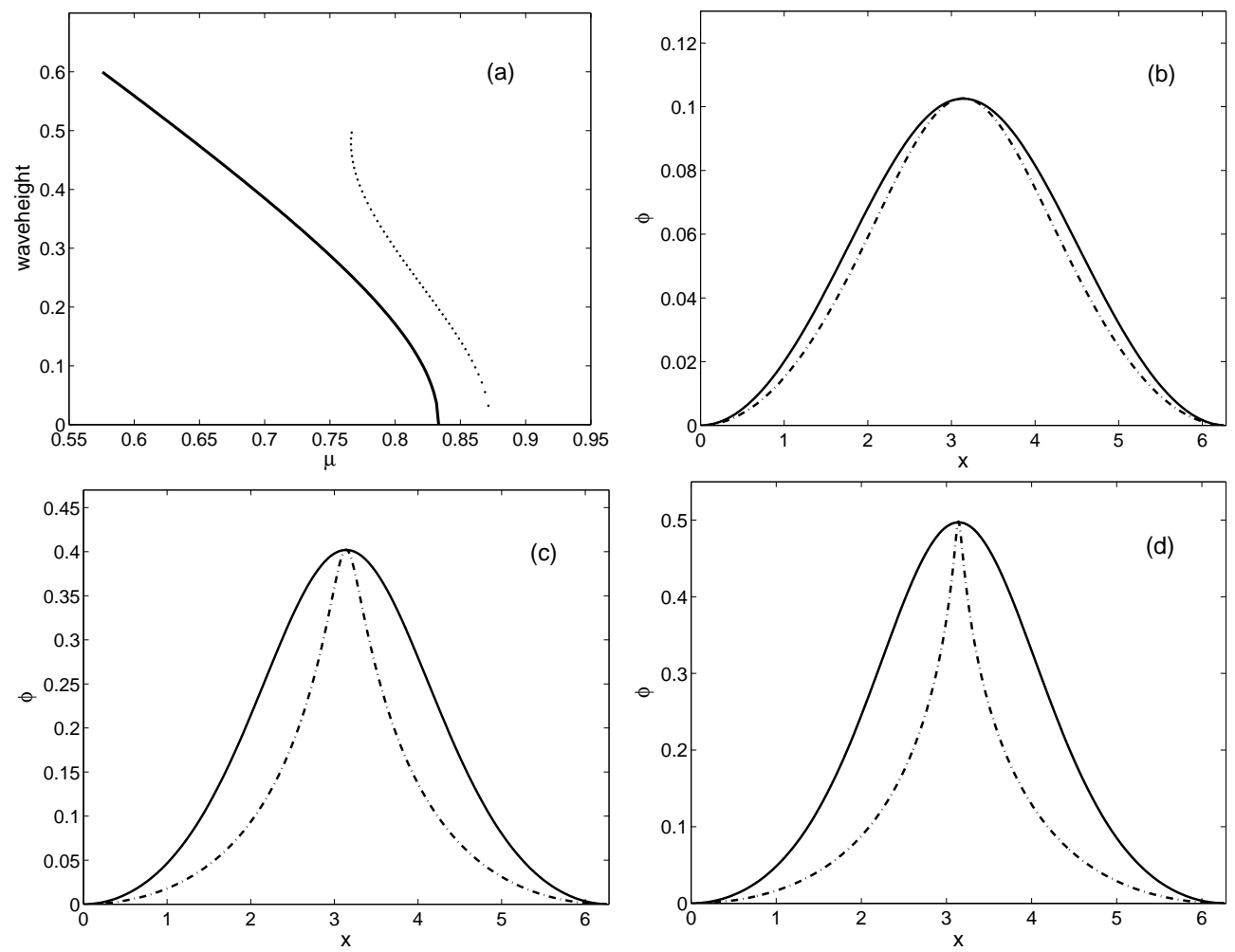

FiguRE 7. A branch of approximate traveling waves for the Whitham equation with $k=1$ (the principal branch) and a branch of traveling waves for the KdV equation are shown in (a). Comparisons between the shapes of Whitham and KdV traveling waves are shown in (b), (c) and (d). The waves are arranged so that the minimum coincides with the $x$-axis. The Whitham waves are represented by dashed-dotted curves, and the $\mathrm{KdV}$ waves are represented by solid curves. The waveheight of the waves is $0.1 \mathrm{in}(\mathrm{b})$, 0.4 in $(\mathrm{c})$ and 0.485 in (d).

Whitham equation. Finally, a comparison was made between the Whitham and KdV traveling waves which revealed that large-amplitude Whitham waves are narrower than their KdV counterparts. It remains to be seen which of these waves resembles the shape of a traveling water wave (the solution of the free-surface boundary-value problem for the Euler equations) more closely.

Acknowledgements. The authors would like to thank Erik Wahlén and Mathew Johnson for constructive comments. This research was supported in part by the Research Council of Norway.

\section{References}

[1] H. Amann. Operator-valued Fourier multipliers, vector-valued Besov spaces, and applications. Math. Nachr., 186 (1997), 5-56.

[2] J. L. Bona, T. Colin, D. Lannes. Long wave approximations for water waves. Arch. Ration. Mech. Anal., 178 (2005), $373-410$.

[3] J. Boyd. Chebyshev and Fourier spectral methods. Dover Publications Inc., Mineola, 2001.

[4] J. P. Boyd. A Legendre-pseudospectral method for computing travelling waves with corners (slope discontinuities) in one space dimension with application to Whitham's equation family. J. Comput. Phys., 189 (2003), 98-110.

[5] B. Buffoni. Existence and conditional energetic stability of capillary-gravity solitary water waves by minimisation. Arch. Ration. Mech. Anal., 173 (2004), 25-68. 
[6] B. Buffoni, J. F. Toland. Analytic theory of global bifurcation. Princeton Series in Applied Mathematics, Princeton University Press, Princeton, 2003.

[7] C. Canuto, M. Y. Hussaini, A. Quarteroni, T.A. Zang. Spectral Methods in Fluid Dynamics. Springer Series in Computational Physics. Springer, New York, 1988.

[8] W. Craig. An existence theory for water waves and the Boussinesq and Korteweg-de Vries scaling limits. Comm. Partial Differential Equations, 10 (1985), 787-1003.

[9] M. Ehrnström, M. D. Groves, E. Wahlén. Solitary waves of the Whitham equation - a variational approach to a class of nonlocal evolution equations and existence of solitary waves of the Whitham equation. Nonlinearity, 25 (2012), 2903-2936.

[10] M. Ehrnström, H. Kalisch. Traveling waves for the Whitham equation. Differential Integral Equations, 22 (2009), 1193-1210.

[11] T. Gneiting. Criteria of Pólya type for radial positive definite functions. Proc. Amer. Math. Soc., 129 (2001), $2309-2318$.

[12] M. D. Groves, E. Wahlén. On the existence and conditional energetic stability of solitary gravity-capillary surface waves on deep water, J. Math. Fluid Mech., 13(4):593-627, 2011.

[13] Y. Katznelson. An introduction to harmonic analysis. Cambridge Mathematical Library, Cambridge University Press, Cambridge, 2004.

[14] H. Kielhöfer. Bifurcation theory. Applied Mathematical Sciences, vol. 156, Springer, New York, 2004.

[15] P. I. Naumkin, I. A. Shishmarev. Nonlinear nonlocal equations in the theory of waves. Translations of Mathematical Monographs. vol. 133. American Mathematical Society, Providence, 1994.

[16] G. Schneider, C. E. Wayne. The long-wave limit for the water wave problem. I. The case of zero surface tension. Comm. Pure Appl. Math., 53 (2000), 1475-1535.

[17] H. Triebel. Theory of function spaces. Monographs in Mathematics, vol. 78, Birkhäuser, Basel, 1983.

[18] G. B. Whitham. Variational methods and applications to water waves. Proc. R. Soc. Lond., A 299 (1967),6-25.

[19] G. B. Whitham. Linear and nonlinear waves. Pure and Applied Mathematics, John Wiley \& Sons, New York, 1974. 